\title{
REVISED MANUSCRIPT
}

\section{GAFF-IC: Realistic Viscosities for Isocyanate Molecules with a GAFF-based Force Field}

\author{
Veniero Lenzi ${ }^{a}$, Piet J. Driest ${ }^{b c}$, Dirk. J. Dijkstra ${ }^{b}$, Luis S. A. Marques ${ }^{a}$ and Marta \\ M. D. Ramos a \\ a Department/Centre of Physics, University of Minho, Campus de Gualtar, 4710-057 Braga, \\ Portugal; ${ }^{\mathrm{b}}$ Covestro Deutschland AG, CAS-Global R\&D, Leverkusen 51365, Germany; ${ }^{\mathrm{c}}$ \\ MIRA Institute for Biomedical Technology and Technical Medicine, Department of \\ Biomaterials Science and Technology, University of Twente, P.O. Box 217, Enschede 7500 \\ AE, The Netherlands
}

\author{
ARTICLE HISTORY \\ Compiled October 25, 2018
}

\begin{abstract}
Aliphatic diisocyanates and their derivatives are key liquid components in the industrial processing of polyurethane materials. In particular, for the synthesis of crosslinked polyurethane materials, the higher functionality molecules obtained by reacting three -or more- diisocyanates are of interest. However, despite their widespread application, the relation between molecular structure and macroscopic physical properties, in particular viscosity, is poorly understood in these systems. In this work, we introduce a new force field parameter set, GAFF-IC, based on the widely-used and versatile GAFF force field, meant for accurate predictions of physical properties of isocyanate-based molecular liquids. The new parameters allow to predict the vaporization enthalpies and densities of several isocyanate-based molecules, which are found in excellent agreement with the available experimental data. The effectiveness and transferability of the improved parameters is verified by calculating the viscosities of several isocyanates, isocyanate dimers(uretdiones) and isocyanate trimers (isocyanurates), resulting in accurate viscosity predictions in excellent agreement with experimental values.
\end{abstract}

\section{KEYWORDS}

Molecular Dynamics, Isocyanates, Isocyanurates, Viscosity, GAFF

\section{Introduction}

Isocyanates are well known[1,2] as the main component in polyurethane (PU) materials, which, due to their versatility, are widely used in diverse applications, from construction blocks to biomaterials. [3,4] Specifically, aliphatic isocyanate trimers (aliphatic isocyanurates), typically obtained by the trimerization reaction of aliphatic diisocyanates, are widely used as building blocks in the synthesis of crosslinked polyurethane materials. The thermal and chemical stability of the isocyanurate-ring $[5,6]$ is one of the reasons behind the properties exhibited by PU materials, such as a high resistance to degradation and good optical,[7,8] mechanical and shape memory properties.[913] Isocyanate themselves have interesting chemical properties, reacting quickly and 
quantitatively at mild conditions with all types of nucleophiles such as alcohols, amines and thiols. For these resasons, isocyanate- and PU chemistry have become a widely studied and applied field of chemistry over the past decades. In recent years, reactions involving isocyanates were also applied in various other fields of research, e.g. for the functionalization of silica surfaces[14-16] and nanocarriers, $[17]$ in modern polymer processing techniques, such as alpha-end polymerisation[18] and post-polymerisation through click-like reactions,[19] in the design of complex surfaces.[20] Furthermore, it was recently shown that aliphatic isocyanates could be obtained through green chemistry routes[21] and in 2015[22] the first commercial bio-based aliphatic polyisocyanate product was introduced to the market, opening new opportunities in the development of sustainable chemistry products.

It is thus expected that aliphatic isocyanates and their derivatives will continue to play a significant role in the design and development of polymeric materials in years to come. It is therefore surprising that in these widely applied systems the connection between molecular structure and macroscopic physical properties, in particular viscosity, remains relatively poorly understood. Although aliphatic isocyanurate products labelled as 'low viscosity' are commercially available, the search for lower viscosity isocyanurates still continues[23,24] $(1000-3000 \mathrm{mPa} \cdot \mathrm{s}$ values are reported for current industrial-grade 1,6-hexamethylene diisocyanate-based isocyanurates). An accurate understanding of the structural and dynamic properties at the molecular scale is required. In this regard, computational techniques, such as molecular dynamics (MD) simulations, offer an invaluable tool to understand the microscopic behaviour of a molecular system and link it to the macroscopic observables. Many molecular dynamics force fields were developed and applied for this purpose, such as CgenFF, [25] ,OPLSAA[26] and GAFF. $[27,28]$ In a previous work, we used MD simulations to study the rheological behaviour of isocyanurate liquids. [29] The GAFF force field was preferred because it was successfully applied on a wide class of systems.[30-36] However, while our simulations were able to reproduce the experimental trends observed for viscosity of isocyanurates, a systematic overestimation of computed values was observed due to the poor description of the intermolecular interactions of these types of molecules in GAFF, since it has been originally devised to reproduce small molecules in water and not pure organic liquids.

The aim of this work is to introduce and test a GAFF-based improved set of parameters for the isocyanate group, for better prediction of the properties of isocyanate and polyisocyanate molecules. This was achieved by modifying the atomic partial charge scheme and the Lennard Jones parameters to best reproduce densities and enthalpies of vaporization of a test set of isocyanate based molecules (figure 1), namely, methyl isocyanate (MIC), Ethyl isocyanate (EIC), Butyl isocyanate (BIC), hexamethylene diisocyanate (HDI), octadecyl isocyanate (ODIC). This set of molecules was chosen in order to span a wide range of molecular weights and includes molecules containing at least one isocyanate group. The choice was also limited by the availability of quality reference data, which is missing for many isocyanates. The simplest molecule in the set is the methyl isocyanate, consisting of an isocyanate group attached to a methyl group. Its geometry is such that no dihedral terms are needed to properly parametrize it, which makes the MIC ideal to study the non-bonded interactions of the NCO group. The newly developed force field was tested by calculating the viscosity of hexamethylene diisocyanate (HDI) and octadecyl isocyanate (ODIC) liquids. Finally, the transferability of the force field for polyisocyanate molecules was tested by calculating the viscosity of three 1,6-hexamethylene diisocyanate (HDI) derivatives: the uretdione (UDI), the symmetrical trimer (3HDI) and the asymmetrical trimer(A3HDI) pure 
liquids.

[Figure 1 about here.]

\section{Methods}

\subsection{Quantum mechanical calculations}

Density functional theory calculations were performed with Gaussian 09 software[37] to obtain optimized geometries and perform relaxed potential energy scans of dihedrals. We adopted the B3LYP hybrid functional,[38] with the empirical dispersion correction terms according to the Grimme's D3 scheme,[39] including the Becke-Johnson[40] damping terms. A triple-zeta $6-311++\mathrm{G}(\mathrm{d}, \mathrm{p})$ basis set,[41] which includes diffuse functions and polarizable orbitals,[42] was used in every calculation.

To derive the partial charges, the structure of each molecule was first optimized at the B3LYP $/ 6-311++\mathrm{G}(\mathrm{d}, \mathrm{p})$ level. Then, the electrostatic potential surface was generated using $\mathrm{HF} / 6-31 \mathrm{G}(\mathrm{d})$ calculations and atomic charges were derived by fitting it following the Restrained Electrostatic Potential (RESP) method.[43]

\subsection{Optimization of the force field parameters}

Equilibrium MD simulations presented in this work were carried out with the LAMMPS package, [44] using the general Amber force field (GAFF) $[27,28]$ to describe the inter- and intramolecular interactions. The energy function employed is given by:

$$
\begin{gathered}
V_{i}=\sum_{i, j}^{\text {bonds }} \frac{k_{b}}{2}\left(r_{i j}-b_{0}\right)^{2}+\sum_{i, j, k}^{\text {angles }} \frac{k_{b}}{2}\left(\theta_{i j k}-\theta_{0}\right)^{2}+\sum_{i, j, k, l}^{\text {dihedrals }} V_{\text {dihe }}\left(\phi_{i j k l}\right)+ \\
\sum_{i, j}^{\text {nonbonded }}\left\{\varepsilon\left[\left(\frac{\sigma_{i j}}{r_{i j}}\right)^{12}-2\left(\frac{\sigma_{i j}}{r_{i j}}\right)^{6}\right]+\frac{1}{4 \pi \epsilon_{0}} \frac{q_{i} q_{j}}{r_{i j}}\right\} \\
r_{i j}=\left|\vec{r}_{i}-\vec{r}_{j}\right|
\end{gathered}
$$

where bond and angle energy terms are described by harmonic potentials, dihedral energies are described by (1), nonbonded interactions consist in a $6-12$ LennardJones (LJ) pair interaction and a standard Coulomb electrostatic term. Non-bonded intramolecular interactions were excluded for atoms involved in the same bond or angle, while for 1-4 atoms in dihedrals, a scaling of 0.5 and 0.833 was adopted for LJ and Coulomb force, respectively.

Antechamber tool from Ambertools 14 utilities [45] was used to extract the atomic charges from RESP calculations and assign standard GAFF parameters to each molecule and generate GAFF topology files.

Isocyanate and isocyanurate group equilibrium bond lengths and angles were adjusted to reproduce the values obtained from B3LYP $/ 6311++\mathrm{G}(\mathrm{d}, \mathrm{p})$ optimized geometries. New LJ parameters for N, C and O atoms of the isocyanate group were obtained 
using the following procedure. The $\varepsilon$ value for the three atoms was initially reduced by $0.055 \mathrm{Kcal} / \mathrm{mol}$ and new parameter sets were defined incrementally considering steps of $0.005 \mathrm{Kcal} / \mathrm{mol}$. Regarding the LJ radii $\sigma_{i j}$, the default values were downsized by subtracting $0.0287 \AA$.

To obtain the fitted molecular mechanics (MM) dihedral parameters, we performed two different scans for every dihedral: one using DFT at B3LYP/6-311++G(d,p) level of theory and the other using MM, excluding any dihedral contribution. We then fitted the difference between the DFT and the MM scan with the following expression:

$$
V_{\text {dihe }}(\phi)=\sum_{i=1}^{4} V_{i}\left[1+\cos \left(n \phi+\delta_{i}\right)\right]
$$

where $\phi$ is the dihedral angle, $V_{i}$ the amplitude and $\delta_{i}$ the phase offset, which can be either 0 or $\pi$. MM calculations were done using Gaussian as well, allowing us to compare directly the different calculations, using $V_{i}$ and $\delta_{i}$ as fitting parameters. The alkyl chain dihedrals were refitted using the HDI torsional energy profiles.

\subsection{Molecular dynamics simulations}

For gas phase simulations, a cubic simulation box of $200 \AA$ length, containing a single molecule, was used without periodic boundary conditions. A very large cutoff for non-bonded interactions was set to avoid any approximation in potential energy calculation and random initial velocities with zero total angular and linear momentum were assigned to all atoms. To calculate the total potential energy, a 25 ns long simulation with a time step of 0.25 fs applying Langevin dynamics with a damping constant of $10 \mathrm{ps}^{-1}$ was performed. Simulation data was sampled every $1.25 \mathrm{ps}$, with the first 300 ps of the simulation were regarded as equilibration and discarded.

For liquid phase simulations of all linear isocyanates and HDI, an initial input file containing one molecule was replicated to obtain a box of 1000 molecules with a very low density, below $0.05 \mathrm{~g} / \mathrm{cm}^{3}$. Boxes with 343 molecules were used for isocyanate trimers and uretdione, and a box of 512 molecule was used for ODIC, to keep the computational cost to an acceptable level. Three independent replicas were considered for each molecular liquid. Subsequently, we assigned random initial velocities corresponding to a temperature of $500 \mathrm{~K}$. To randomize the initial structures, atom coordinates were scaled by 0.8 and a short run of 1 ps with a 1 fs time step has been performed using NVE conditions with a constrained maximum displacement per step per atom to $0.1 \AA$. A cutoff of $13 \AA$ for LJ interaction was set and no electrostatic interactions were considered. This scale-and-run step is repeated until a density of 0.7 $\mathrm{g} / \mathrm{cm}^{3}$ is reached. For larger molecules, we performed a supplementary 500 ps run at $500 \mathrm{~K}$ and $1 \mathrm{~atm}$, to further randomize the molecules positions and conformations. Next, 1 ns equilibration runs under NPT conditions were performed, with 1 fs time step, using a Noose-Hoover[46,47] thermo- and barostat with relaxation times of 0.1 and $1 \mathrm{ps}$, respectively. To ensure a good description of the fastest degrees of freedom of the molecules and a good total energy conservation, a rRESPA[48,49] algorithm was applied, with an inner time step of 0.125 fs. Direct calculation of all pair forces was limited by a cutoff of $13 \AA$, and the Particle-Particle-Particle Mesh (P3M)[49,50] method was used for the long-range contribution of Coulomb interactions. The P3M method was applied as well to treat long-range dispersion interactions. [51] To verify the onset of equilibrium conditions, the total energy of was monitored and, if needed, 
the equilibration time was prolonged until stationarity in total energy was achieved(see Figure SI.1 of supplementary information). Typical production runs from equilibrated states were carried on for 5 ns and the quantities of interest printed out every 100 fs. All the presented quantities are the result of an ensemble average over the production run and over the independent replicas, and their errors were estimated from fluctuations in ensemble average: $\Delta_{X}=\sqrt{(\langle X-\langle X\rangle\rangle)^{2}}$.

Vaporization enthalpies were calculated using the following equation:[52]

$$
\Delta H_{\text {vap }}=U_{\text {gas }}-U_{\text {liq }}+R T+\frac{1}{2} R\left(\left\langle T_{\text {liq }}\right\rangle-\left\langle T_{\text {gas }}\right\rangle\right)\left(3 N_{\text {atoms }}-6\right)
$$

where $T$ is the system target temperature, $U_{\text {gas }}$ and $U_{\text {liq }}$ the potential energy per molecule for the gas and the liquid phase, respectively. The last term appearing in (2) is a correction term that takes into account the difference between the gas phase temperature $\left\langle T_{\text {gas }}\right\rangle$ and the liquid phase temperature $\left\langle T_{\text {liq }}\right\rangle$ extracted from the simulation.

For viscosity calculation, simulations were performed in NVE conditions, starting from equilibrium states at the desired temperature and pressure, with thermodynamic data and the 6 components of the symmetric traceless pressure tensor $s_{i j}$ saved every $d t=3$ fs. We used the Green-Kubo[53] formula, which was preferred to nonequilibrium methods because it provides directly the zero-shear viscosity. It involves the autocorrelation function of all components of the symmetric traceless pressure tensor components $s_{i j}:[54]$

$$
\eta=\frac{V}{10 k_{b} T}\left\langle\int_{t^{\prime}}^{t^{\prime}+t_{W}} s_{i j}\left(t+t^{\prime}\right) s_{i j}\left(t^{\prime}\right) \mathrm{d} t\right\rangle
$$

where angular brackets indicate average over different possible time origins $t^{\prime}$, separated by $t_{s}=15 \mathrm{fs}$, used for all viscosity calculations. Whereas an integration time window $t_{W}$ of $50 \mathrm{ps}$ was sufficient to ensure the convergence of symmetric traceless pressure tensor components correlation integral for the low-viscosity systems, namely all isocyanates and HDI, a larger $t_{W}$ was considered for isocyanate dimers and trimers, up to $t_{W}=600 \mathrm{ps}$. The uncertainty on viscosity was estimated calculating, for each run, the standard deviation of the converged values of the autocorrelation integral of each of the symmetric traceless pressure tensor components.

\subsection{Materials and procedures}

All unreferenced experimental data were obtained using materials and procedures described in [29]. Density measurements were performed using a pycnometer. The enthalpy of vaporization of HDI was calculated using the Clausius-Clapeyron equation as an average from vapour pressures in the range of $393.15-493.15 \mathrm{~K}$, which were determined ebulliometrically. Octadecyl isocyanate (ODIC) and HDI uretdione (UDI) were isolated starting from octadecylamine (purchased from Sigma-Aldrich) and industrial-grade HDI-Uretdione (Desmodur N3400), respectively. The density of the asymmetrical HDI trimer (A3HDI) was assumed to be equal to pure 3HDI[29] and its viscosity was estimated by measuring the viscosity of varius mixtures of pure 3HDI and the industrial grade A3HDI/3HDI mixture Desmodur N3900 at different ratios, and subsequent extrapolation to pure A3HDI. 


\section{Results and discussion}

\subsection{Optimization of non-bonded parameters and torsional potentials}

The density $\rho$ and vaporization enthalpy $\Delta H_{v a p}$ of a MIC pure liquid were obtained at simulation conditions of $293.15 \mathrm{~K}$ and $1 \mathrm{~atm}$, using different choices for non-bonded parameters. When compared against the reference values listed in table 1 , the unoptimized GAFF overestimates density and vaporization enthalpy by $8 \%$ and $42 \%$ respectively, in line with what was reported elsewhere, $[32,33,35]$ indicating that liquid cohesive forces are probably exaggerated by GAFF.

One way to correct this overestimation, is by scaling the atomic charges as done in the MDEC model for simulations of ionic liquids[31,55] with a scaling factor of $\frac{1}{\sqrt{2}}$ being adopted. The charge-scaling improves the agreement between calculations and experiments, however still overestimating the reference values, with a density of $1.001 \pm 0.005 \mathrm{~g} / \mathrm{cm}^{3}$ and a vaporization enthalpy of $37.6 \pm 6.5 \mathrm{~kJ} / \mathrm{mol}$. To test whether a stronger scaling could cure this behaviour, a scaling factor of $\frac{1}{\sqrt{10}}$ was also considered, but little improvement was observed with respect to the previous scaling, with a $\rho$ of $0.989 \pm 0.005$ and a $\Delta H_{\text {vap }}$ of $39.9 \pm 6.8$. This means that the charge scaling alone is not sufficient to achieve good accuracy, indicating that NCO-NCO interactions, apart from a significant electrostatic contribution, contain a relevant dispersion part, hence the isocyanate N,C and O LJ parameters should be optimized as well.

In this regard, the LJ parameters adopted for alkyl carbons and related hydrogens are those proposed by Dickson et al.[33] while, for NCO-group atoms, different parameter sets were tested and the best result was obtained with the GAFF-IC parameter set (see supplementary information), which provided an excellent agreement with both density and vaporization enthalpy with deviations better than $1 \%$ and $10 \%$, respectively.

Standard GAFF is known for poorly reproducing the dihedral energies of alkanes,[33] which affects its predictions of liquid phase properties. Figure 2 shows the dihedral energy curves obtained from the optimized parameters. The overall accuracy increased significantly with respect to the standard GAFF, with an almost perfect match obtained for C-C-C-C dihedrals, while the C-C-C-N was greatly improved, especially the minima at \pm 60 degrees.

[Figure 2 about here.]

\subsection{Testing the GAFF-IC force field}

Table 1 lists the molecule set considered for GAFF-IC testing, along with the experimental and calculated values for density, vaporization enthalpy and viscosity.

[Table 1 about here.]

[Figure 3 about here.]

[Figure 4 about here.]

The calculated and experimental values of $\rho$ and $\Delta H_{\text {vap }}$ for the molecules in table 1 , are plotted in figures 3 and 4 , respectively. The densities calculated with standard GAFF are slightly overestimated, with a relative mean absolute deviation (MAD) of 
$2.79 \%$ while GAFF-IC predicts densities very close to the reference ones, with a relative MAD of $0.748 \%$. Concerning vaporization enthalpies, the use of GAFF resulted in a marked overestimation of $\Delta H_{v a p}$ for all the molecules considered, producing a MAD of $55.92 \%$. Conversely, the GAFF-IC predicted $\Delta H_{\text {vap }}$ values are much closer to the experimental ones, with a MAD of $12.01 \%$. An exception seems to be represented by the HDI and ODIC, for which the GAFF-IC force field overestimates $\Delta H_{\text {vap }}$ by $17.5 \%$ and $17.7 \%$, respectively. However, the reference values lie within the uncertainty of the GAFF-IC prediction, and it clearly represents an improvement with respect to the unoptimized parameters, that overestimated the experimental $\Delta H_{v a p}$ by a factor 2 .

[Figure 5 about here.]

[Figure 6 about here.]

The ability of GAFF-IC to predict the dynamic properties of isocyanate-based systems was also tested by calculating the viscosity of liquid isocyanates and isocyanurates and comparing them with the experimental data available. Experimental and calculated viscosity values are reported in figures 5 . Due to the high viscosity at room temperature of isocyanate trimers, [29] Green-Kubo calculations failed to provide converged results. Therefore, viscosity measurements for trimers were performed at higher temperatures to allow a direct comparison between experiments and simulations. The standard GAFF force field systematically overestimates viscosities, with HDI and 3HDI viscosities overestimated by about one order of magnitude and giving larger values for the smaller isocyanates (MIC, EIC, BIC), when compared with GAFF-IC results. In turn, GAFF-IC viscosity predictions almost match experimental values. Concerning polyisocyanates, our results show once again a large difference between the GAFF and GAFF-IC, with standard GAFF even failing to provide converged values. On the other hand, GAFF-IC provides very accurate estimates of viscosities for 3HDI and UDI, in excellent agreement with experimental values, and reproduces very nicely the expected A3HDI/3HDI viscosity ratio.

Figure 6 reports a comparison between experimental and calculated viscosities for the 3HDI trimer at three different temperatures. An excellent agreement with experimental data was found, with the predicted viscosity of $22.9 \pm 6.8 \mathrm{mPa} \cdot \mathrm{s}$ at $378.15 \mathrm{~K}$ and $12.6 \pm 1.2 \mathrm{mPa} \cdot \mathrm{s}$ at $398.15 \mathrm{~K}$ matching the experimental measurements at the corresponding temperature of $17.6 \mathrm{mPa} \cdot \mathrm{s}$ and $12.3 \mathrm{mPa} \cdot \mathrm{s}$. This shows that the GAFF-IC parameters provide reliable viscosity predictions even at different temperatures with respect to the one used for its parametrisation.

The newly introduced parametrization greatly improved the prediction of the addressed quantities for isocyanate-based systems, and could serve as a basis for coarsegrained models. In this sense, further research of our group will focus on further developments of GAFF-IC to expand its applicability to study isocyanate-based polymer mixtures, as well as the interaction of isocyanates with different surfaces.

\section{Conclusions}

The GAFF-IC forcefield was developed starting from standard GAFF, and correctly predicted the densities and vaporization enthalpies of isocyanate-based molecular liquids, providing a 5-fold increase in accuracy for both quantities, when compared with GAFF. The use of GAFF-IC for viscosity calculations resulted in a striking impro- 
vement of viscosity predictions. Excellent agreement with experimental data was found for HDI molecule, curing the observed order-of-magnitude overestimations of GAFF calculations. Although it was parametrized starting from monoisocyanates, GAFF-IC also proved to be transferable to polyisocyanate molecules, providing viscosities in excellent agreement with experiments as well. Our study demonstrates that GAFF-IC is a powerful tool to reliably predict densities, vaporization enthalpies and viscosities of isocyanurate materials and hence help in the design of new processing routes and materials based on isocyanates.

\section{Acknowledgements}

The authors wish to thank Dr. Frank Richter and his research team at Covestro Deutschland AG for continued collaboration and fruitful discussions.

\section{Disclosure statement}

There are no conflicts of interest to declare.

\section{Funding}

This project has received funding from the European Union's Horizon 2020 research and innovation program under the Marie Skłodowska-Curie Grant Agreement no. 642890 (http://thelink-project.eu/) and it was partially supported by the Portuguese Foundation for Science and Technology (FCT) in the framework of the Strategic Funding UID/FIS/04650/2013, and by the project "Search-ON2: Revitalization of HPC infrastructure of Uminho" (NORTE-07-0162-FEDER-0000869), under the National Strategic Reference Framework, through the European Regional Development Fund.

\section{References}

[1] Wurtz A. Recherches sur les thers cyaniques et leurs drivs. Comptes Rendu Chimie. 1848; 27:241-243.

[2] Bayer O. Das di-isocyanat-polyadditionsverfahren (polyurethane). Angewandte Chemie. 1947;59(9):257-272. Available from: http://dx.doi.org/10.1002/ange.19470590901.

[3] Sonnenschein MF. Polyurethanes, science, technology, markets, and trends. Hoboken, NJ, USA: John Wiley and Sons; 2015.

[4] Cooper SL, Guan J, editors. Advances in polyurethane biomaterials. Woodhead Publishing; 2016.

[5] Heift D, Benko Z, Grutzmacher H, et al. Cyclo-oligomerization of isocyanates with na(ph2) or na(ocp) as "p-" anion sources. Chem Sci. 2015;6:4017-4024. Available from: http: //dx.doi.org/10.1039/C5SC00963D.

[6] Okumoto S, Yamabe S. A computational study of base-catalyzed reactions between isocyanates and epoxides affording 2-oxazolidones and isocyanurates. Journal of Computational Chemistry. 2001;22(3):316-326. Available from: http://dx.doi.org/10.1002/ 1096-987X (200102) 22:3<316: :AID-JCC1004>3. 0. C0;2-5.

[7] Flipsen TAC, Steendam R, Pennings AJ, et al. A novel thermoset polymer optical fiber. Advanced Materials. 1996;8(1):45-48. Available from: http://dx.doi.org/10. 1002/adma. 19960080107. 
[8] Fabbri P, Poor SM, Ferrari L, et al. Highly stable plastic optical fibre amplifiers containing [eu(btfa)3(meoh)(bpeta)]: A luminophore able to drive the synthesis of polyisocyanates. Polymer. 2014;55(2):488 - 494. Available from: http://www.sciencedirect.com/ science/article/pii/S0032386113011439.

[9] Chattopadhyay D, Raju K. Structural engineering of polyurethane coatings for high performance applications. Progress in Polymer Science. 2007;32(3):352 - 418. Available from: http://www.sciencedirect.com/science/article/pii/S0079670006001365.

[10] Moritsugu M, Sudo A, Endo T. Cyclotrimerization of diisocyanates toward highperformance networked polymers with rigid isocyanurate structure: Combination of aromatic and aliphatic diisocyanates for tunable flexibility. Journal of Polymer Science Part A: Polymer Chemistry. 2013;51(12):2631-2637. Available from: http://dx.doi.org/10. 1002/pola. 26651.

[11] Renz H, Bruchmann B. Pathways targeting solvent-free $\{$ PUR $\}$ coatings. Progress in Organic Coatings. 2001;43(13):32 - 40. Athens 2000; Available from: http://www. sciencedirect.com/science/article/pii/S0300944001002399.

[12] Preis E, Schindler N, Adrian S, et al. Microporous polymer networks made by cyclotrimerization of commercial, aromatic diisocyanates. ACS Macro Letters. 2015;4(11):1268-1272. Available from: http://dx.doi.org/10.1021/acsmacrolett.5b00726.

[13] Donthula S, Mandal C, Leventis T, et al. Shape memory superelastic poly(isocyanurateurethane) aerogels (pir-pur) for deployable panels and biomimetic applications. Chemistry of Materials. 2017;29(10):4461-4477. Available from: http://dx .doi.org/10.1021/acs . chemmater. 7b01020.

[14] Kang HJ, Cha EJ, Park HD. Protein immobilization onto various surfaces using a polymer-bound isocyanate. Applied Surface Science. 2015;324(Supplement C):198 - 204. Available from: http://www.sciencedirect.com/science/article/pii/ S0169433214023605.

[15] Howarter J, Youngblood J. Self-cleaning and anti-fog surfaces via stimuli-responsive polymer brushes. Advanced Materials. 2007;19(22):3838-3843. Available from: http: //dx.doi.org/10.1002/adma.200700156.

[16] McDonald AR, Dijkstra HP, Suijkerbuijk BMJM, et al. click immobilization of organometallic pincer catalysts for cc coupling reactions. Organometallics. 2009;28(16):4689-4699. Available from: http://dx.doi.org/10.1021/om900237g.

[17] Kuypers S, Pramanik SK, D'Olieslaeger L, et al. Interfacial thiol-isocyanate reactions for functional nanocarriers: a facile route towards tunable morphologies and hydrophilic payload encapsulation. Chem Commun. 2015;51:15858-15861. Available from: http:// dx.doi.org/10.1039/C5CC05258K.

[18] Gody G, Rossner C, Moraes J, et al. One-pot raft/click chemistry via isocyanates: Efficient synthesis of -end-functionalized polymers. Journal of the American Chemical Society. 2012;134(30):12596-12603. PMID: 22731785; Available from: http://dx.doi.org/10. 1021/ja3030643.

[19] Gody G, Roberts DA, Maschmeyer T, et al. A new methodology for assessing macromolecular click reactions and its application to aminetertiary isocyanate coupling for polymer ligation. Journal of the American Chemical Society. 2016;138(12):4061-4068. PMID: 26927624; Available from: http://dx.doi.org/10.1021/jacs.5b11831.

[20] Arnold RM, Patton DL, Popik VV, et al. A dynamic duo: Pairing click chemistry and postpolymerization modification to design complex surfaces. Accounts of Chemical Research. 2014;47(10):2999-3008. PMID: 25127014; Available from: http://dx.doi.org/10. $1021 / \operatorname{ar} 500191 \mathrm{~m}$.

[21] Sahoo S, Kalita H, Mohanty S, et al. Synthesis and characterization of vegetable oil based polyurethane derived from low viscous bio aliphatic isocyanate: Adhesion strenghth to to wood-wood substrate bonding. Macromolecular Research. 2017;25(8):772-778.

[22] Behnken G, Hecking A, Sánchez BV. High performance enabled by nature. Eur Couatings Journal. 2016;1:46-50.

[23] Ludewig M, Weikard J, Stockel N. Allophanate-containing modified polyurethanes ; 
2006. US Patent App. 11/369,483; Available from: https://www.google.com/patents/ US20060205911.

[24] Dieris C, Mertes H, Pedain J, et al. Isocyanattrimerisate und isocyanattrimerisatmischungen, deren herstellung und verwendung ; 2003. EP Patent 0,798,299; Available from: https : //www.google.com/patents/EP0798299B1?cl=it.

[25] Vanommeslaeghe K, Hatcher E, Acharya C, et al. Charmm general force field: A force field for drug-like molecules compatible with the charmm all-atom additive biological force fields. Journal of Computational Chemistry. 2010;31(4):671-690. Available from: http://dx.doi.org/10.1002/jcc.21367.

[26] Jorgensen WL, Maxwell DS, Tirado-Rives J. Development and testing of the opls all-atom force field on conformational energetics and properties of organic liquids. Journal of the American Chemical Society. 1996;118(45):11225-11236.

[27] Cornell WD, Cieplak P, Bayly CI, et al. A second generation force field for the simulation of proteins, nucleic acids, and organic molecules. Journal of the American Chemical Society. 1995;117(19):5179-5197.

[28] Wang J, Wolf RM, Caldwell JW, et al. Development and testing of a general amber force field. Journal of Computational Chemistry. 2004;25(9):1157-1174. Available from: http://dx.doi.org/10.1002/jcc. 20035.

[29] Driest PJ, Lenzi V, Marques LSA, et al. Aliphatic isocyanurate and polyisocyanate networks. Polymers for advanced technologies. 2017;28(10):1299-1304. Available from: http://dx.doi.org/10.1002/pat.3891.

[30] Sprenger KG, Jaeger VW, Pfaendtner J. The general amber force field (gaff) can accurately predict thermodynamic and transport properties of many ionic liquids. The Journal of Physical Chemistry B. 2015;119(18):5882-5895. PMID: 25853313; Available from: http://dx.doi.org/10.1021/acs.jpcb.5b00689.

[31] Verevkin SP, Zaitsau DH, Emel'Yanenko VN, et al. Making Sense of Enthalpy of Vaporization Trends for Ionic Liquids: New Experimental and Simulation Data Show a Simple Linear Relationship and Help Reconcile Previous Data. Journal of Physical Chemistry B. 2013;117(21):6473-6486.

[32] Caleman C, van Maaren PJ, Hong M, et al. Force field benchmark of organic liquids: Density, enthalpy of vaporization, heat capacities, surface tension, isothermal compressibility, volumetric expansion coefficient, and dielectric constant. Journal of Chemical Theory and Computation. 2012;8(1):61-74.

[33] Dickson CJ, Rosso L, Betz RM, et al. GAFFlipid: a General Amber Force Field for the accurate molecular dynamics simulation of phospholipid. Soft Matter. 2012;8:9617.

[34] Dickson CJ, Madej BD, Skjevik ÅA, et al. Lipid14: The Amber Lipid Force Field. Journal of chemical theory and computation. 2014;10(2):865-879. Available from: http://dx. doi.org/10.1021/ct4010307.

[35] Boyd NJ, Wilson MR. Optimization of the GAFF force field to describe liquid crystal molecules: the path to a dramatic improvement in transition temperature predictions. Physical Chemistry Chemical Physics. 2015;17(38):24851-24865. Available from: http: //xlink.rsc.org/?DOI=C5CP03702F.

[36] Ozpinar GA, Peukert W, Clark T. An improved generalized AMBER force field (GAFF) for urea. Journal of Molecular Modeling. 2010;16(9):1427-1440.

[37] Frisch MJ, Trucks GW, Schlegel HB, et al. Gaussian '09 Revision E.01 ; 2009. Gaussian Inc. Wallingford CT.

[38] Becke AD. Density-functional thermochemistry. iii. the role of exact exchange. The Journal of Chemical Physics. 1993;98(7):5648-5652. Available from: http://scitation.aip. org/content/aip/journal/jcp/98/7/10.1063/1.464913.

[39] Grimme S, Antony J, Ehrlich S, et al. A consistent and accurate ab initio parametrization of density functional dispersion correction (dft-d) for the 94 elements h-pu. The Journal of Chemical Physics. 2010;132(15):154104. Available from: http://scitation.aip.org/ content/aip/journal/jcp/132/15/10.1063/1.3382344.

[40] Grimme S, Ehrlich S, Goerigk L. Effect of the damping function in dispersion corrected 
density functional theory. Journal of Computational Chemistry. 2011;32(7):1456-1465. Available from: http://dx.doi.org/10.1002/jcc.21759.

[41] Ditchfield R, Hehre WJ, Pople JA. Self-consistent molecular-orbital methods. ix. an extended gaussian-type basis for molecular-orbital studies of organic molecules. The Journal of Chemical Physics. 1971;54(2):724-728. Available from: http://scitation.aip.org/ content/aip/journal/jcp/54/2/10.1063/1.1674902.

[42] Clark T, Chandrasekhar J, Spitznagel GW, et al. Efficient diffuse function-augmented basis sets for anion calculations. iii. the $3-21+\mathrm{g}$ basis set for first-row elements, li-f. Journal of Computational Chemistry. 1983;4(3):294-301. Available from: http://dx.doi.org/ 10.1002/jcc.540040303.

[43] Bayly CI, Cieplak P, Cornell W, et al. A well-behaved electrostatic potential based method using charge restraints for deriving atomic charges: the resp model. The Journal of Physical Chemistry. 1993;97(40):10269-10280. Available from: http://dx.doi.org/10. $1021 / \mathrm{j} 100142 \mathrm{a} 004$.

[44] Plimpton S. Fast parallel algorithms for short-range molecular dynamics. Journal of Computational Physics. 1995;117(1):1 - 19. Available from: http://www.sciencedirect. com/science/article/pii/S002199918571039X.

[45] Case D, Betz R, Botello-Smith W, et al. ; 2016. AMBER 2016, University of California, San Francisco, CA, USA.

[46] Hoover WG. Canonical dynamics: Equilibrium phase-space distributions. Phys Rev A. 1985 Mar;31:1695-1697. Available from: http://link.aps.org/doi/10.1103/ PhysRevA.31.1695.

[47] Parrinello M, Rahman A. Polymorphic transitions in single crystals: A new molecular dynamics method. Journal of Applied Physics. 1981;52(12):7182-7190. Available from: http://scitation.aip.org/content/aip/journal/jap/52/12/10.1063/1.328693.

[48] Tuckerman M, Berne BJ, Martyna GJ. Reversible multiple time scale molecular dynamics. The Journal of Chemical Physics. 1992;97(3):1990-2001. Available from: http: //scitation.aip.org/content/aip/journal/jcp/97/3/10.1063/1.463137.

[49] Plimpton SJ, Pollock R, Stevens M. Particle-mesh ewald and rrespa for parallel molecular dynamics simulations. In: PPSC; 1997.

[50] Hockney RW, Eastwood JW. Computer simulation using particles. Bristol, PA, USA: Taylor \& Francis, Inc.; 1988.

[51] Isele-Holder RE, Mitchell W, Ismail AE. Development and application of a particleparticle particle-mesh ewald method for dispersion interactions. The Journal of Chemical Physics. 2012;137(17):174107. Available from: https://doi.org/10.1063/1.4764089.

[52] Wang J, Hou T. Application of molecular dynamics simulations in molecular property prediction. 1. density and heat of vaporization. Journal of Chemical Theory and Computation. 2011;7(7):2151-2165. PMID: 21857814; Available from: http://dx.doi.org/10. $1021 /$ ct200142z.

[53] Hansen JP, Macdonald IR. Theory of simple liquids. 4th ed. Oxford, UK: Academic Press; 2013.

[54] Daivis PJ, Evans DJ. Comparison of constant pressure and constant volume nonequilibrium simulations of sheared model decane. The Journal of Chemical Physics. 1994; 100(1):541-547. Available from: http://scitation.aip.org/content/aip/journal/ jcp/100/1/10.1063/1.466970.

[55] Leontyev I, Stuchebrukhov A. Accounting for electronic polarization in non-polarizable force fields. Physical Chemistry Chemical Physics. 2011;13(7):2613-2626.

[56] Berger LI, Frenkel M, Koh CA, et al. CRC Handbook of Chemistry and Physics. 95th ed. Boca Raton, FL, USA: CRC Press; 2014.

[57] Chickos JS, Acree WE. Enthalpies of vaporization of organic and organometallic compounds, 1880-2002. Journal of Physical and Chemical Reference Data. 2003;32(2):519878.

[58] Cheméo - high quality chemical properties ; 2018. Available from: www.chemeo.com.

[59] Chemspider - search and share chemistry ; 2018. Available from: www.chemspider.com. 
[60] European chemicals agency ; 2018. Available from: https://echa. europa.eu. 


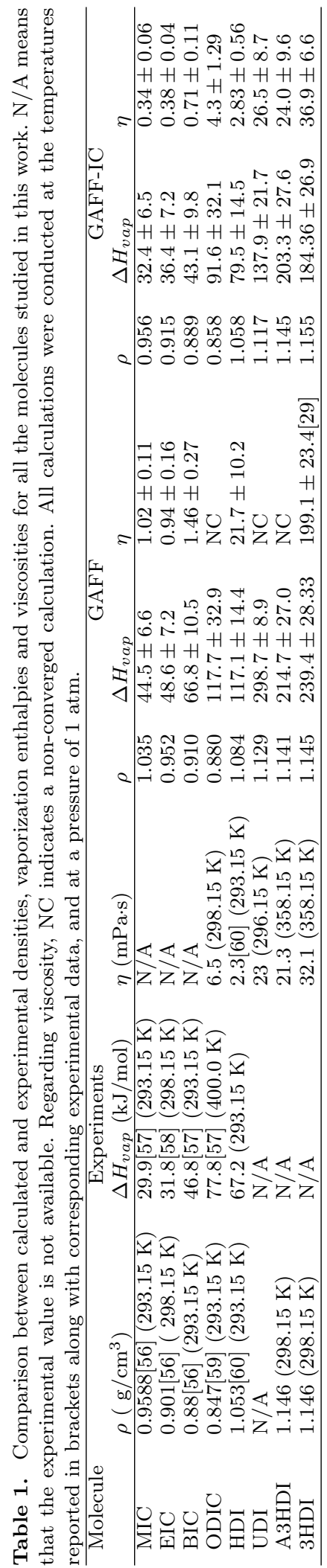




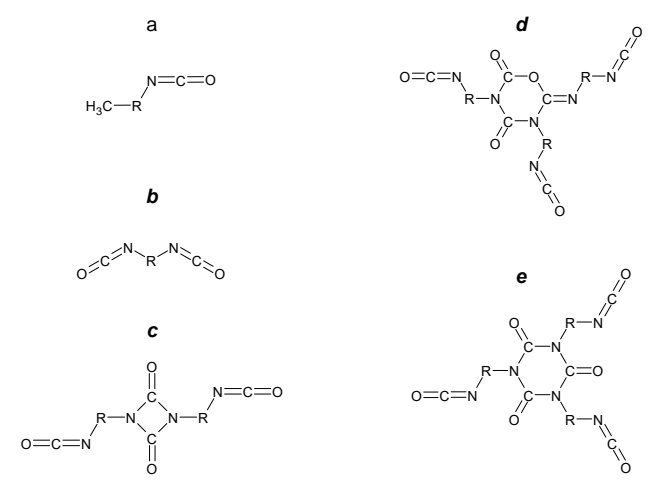

Figure 1. Overview of aliphatic isocyanates considered in this work. In all formulas, $\mathrm{R}$ represents an alkylene group: $\mathrm{R}=-\left(\mathrm{CH}_{2}\right)_{\mathrm{n}}-$. Monoisocyanates such as MIC, BIC, EIC and ODIC have the same structure as (a), differing by the number of carbons in the alkylene group $\mathrm{R}$. The general structure of diisocyanates is represented in (b), where for HDI $\mathrm{R}=-\left(\mathrm{CH}_{2}\right)_{6}-$. Uretdiones, or isocyanate dimers (c), and the asymmetrical (d) and symmetrical (e) form of isocyanate trimers are represented as well. 


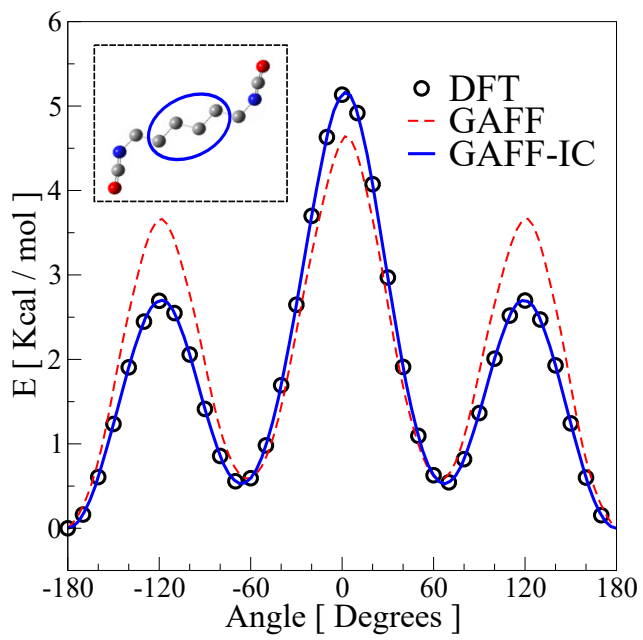

(a)

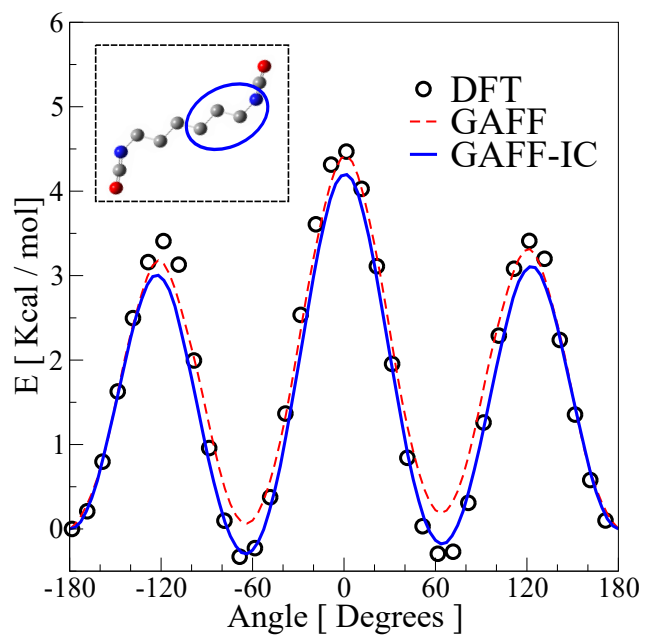

(b)

Figure 2. Comparison between B3LYP/6-311 $++\mathrm{g}(\mathrm{d}, \mathrm{p})$ (circles) and fitted molecular mechanics parameters dihedral energies (lines) for a central C-C-C-C dihedral (a) and a terminal C-C-C-N dihedral (b), as shown in insets. Mean absolute deviation is $0.2 \mathrm{Kcal} / \mathrm{mol}$. 


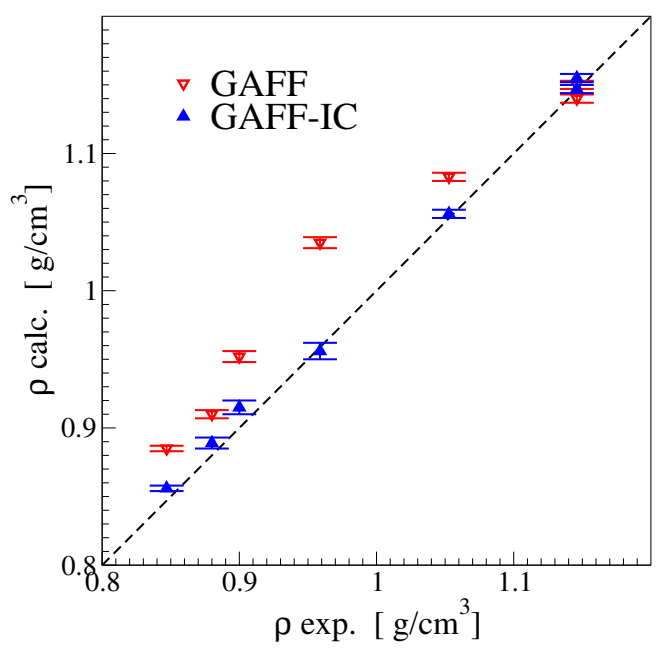

Figure 3. Deviation against experimental values of the calculated $\rho$ of all the molecules of table 1. Plots are restricted to those molecules for which measurements were available. Dashed line is $y=x$. 


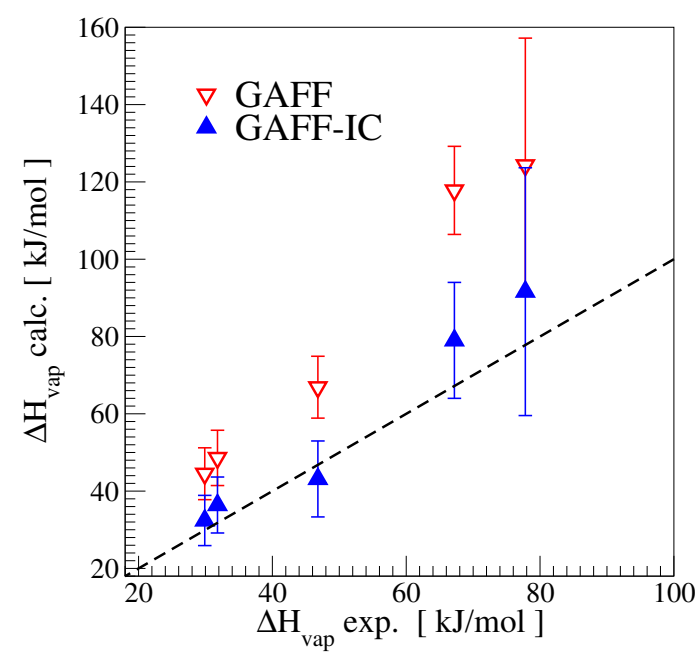

Figure 4. Deviation against experimental values of the calculated $\Delta H_{v a p}$ of all the molecules of table 1 . Plots are restricted to those molecules for which measurements were available. Dashed line is $y=x$. 


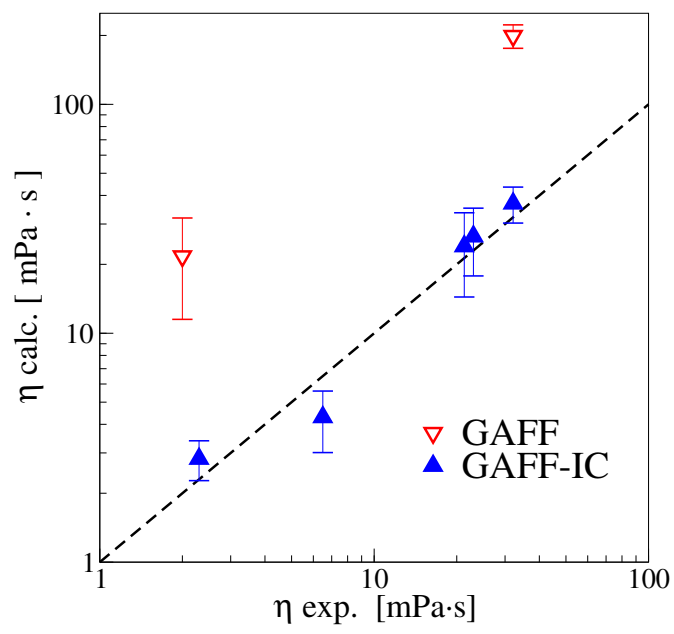

Figure 5. Deviation against experimental values of the calculated $\eta$ of all the molecules of table 1, and calculated at the temperatures reported therein. The plot is restricted to those molecules for which measurements were available. Dashed line is $y=x$. The scale is logarithmic for both axes. 


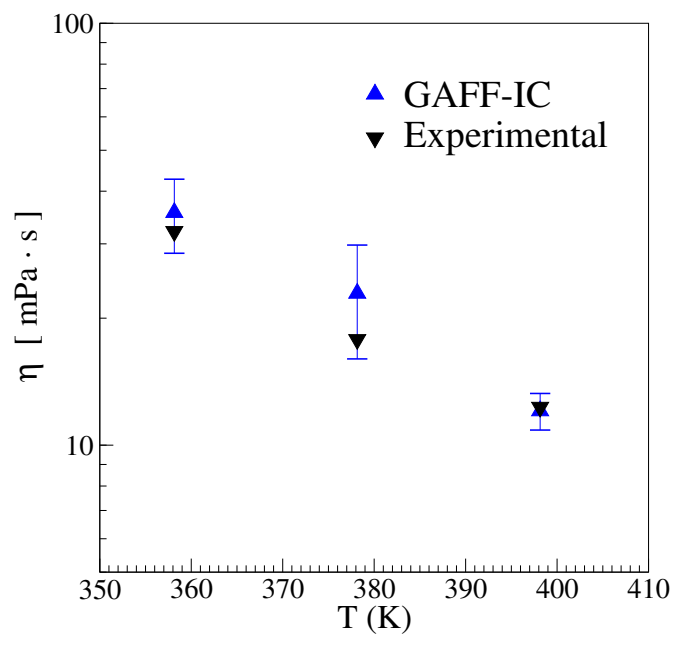

Figure 6. Comparison between experimental and calculated viscosities for a pure 3HDI trimer liquid, at different temperatures. The ordinate scale is logarithmic. 